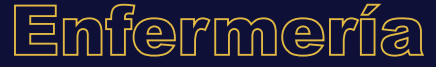

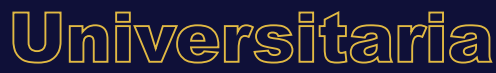

\section{Conocimientos, actitudes y motivaciones respecto a la afiliación al colegio de enfermeras en docentes universitarios}

\section{Knowledge, attitudes, and motivations among university teachers regarding the affiliation to a Chilean College of Nurses}

\section{Conhecimentos, atitudes e motivações ao respeito da filiação ao colégio de enfermeiras em docentes universitários}

\author{
C.A. Fernández-Silva ${ }^{\mathrm{a}^{*}}$, M.I. Riquelme-Rivera ${ }^{\mathrm{b} 2}$, \\ C.A. Cannobbio-Chiguay ${ }^{\mathrm{c}}$, B. Pilquina $o^{\mathrm{d} 4}$ \\ ORCID:

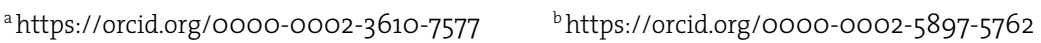 \\ c https://orcid.org/00oo-0001-6641-4319 ${ }^{\mathrm{d}}$ https://orcid.org/0ooo-0002-596o-6344

\begin{abstract}
'Universidad de los Lagos, Departamento de Salud, Puerto Montt, Chile ${ }^{2}$ Universidad Austral de Chile, Escuela de enfermería, Sede Puerto Montt, Chile

3Universidad San Sebastián, Carrera de Enfermería, Sede Patagonia, Chile ${ }^{4}$ Universidad Santo Tomás, Sede Puerto Montt, Chile

Recibido: 9 julio 2019 / Aceptado: 10 diciembre 2019
\end{abstract}

RESUMEN

Introducción: La participación en el colegio de enfermeras es una instancia relevante para el desarrollo de la profesión a nivel país, debido a la defensa que realiza por la enfermería desde los diferentes ámbitos de actuación; es indispensable inculcar esta visión desde la formación universitaria a través de los profesionales de enfermería que se dedican a la docencia. Materiales y métodos: Se realizó un estudio cuantitativo, transeccional y correlacional, en 49 académicos de las diferentes casas de estudio de la ciudad de Puerto Montt-Chile, en quienes se evaluó su afiliación al colegio de enfermeras, además de sus conocimientos, actitudes y motivaciones respecto a participar en dicha instancia gremial; para tal fin se aplicó un instrumento de elaboración propia ( $\alpha$ Cronbach 0.71).

Resultados: Se encontró que el 40.9\% de los docentes está afiliado al colegio de enfermeras y que existe conocimiento suficiente respecto a esta organización, con una actitud

*Autor de correspondencia. Correo electrónico: carlosalberto.fernandez@ulagos.cl 
desfavorable hacia el mismo, evidenciándose asociación entre la actitud y aspectos como la dinámica de trabajo ( $p=0.043$ Correlación de Spearman: 0.158 ) y la afiliación al colegio ( $p=0.02$ Coeficiente de Spearman: 0.142).

Discusión: La participación colegiada permite el fortalecimiento de la profesión, además de favorecer la visibilidad, liderazgo e identidad profesional; aunque se reconocen estos aspectos no son suficientes para generar la afiliación o una actitud favorable a ésta.

Conclusiones: En la promoción de la afiliación debe considerarse la tendencia a no afiliación de los docentes más jóvenes y los aspectos de motivación, ambos relevantes para destacar la participación colegial como un aspecto deontológico que forma parte del modelaje hacia los enfermeros en formación.

Palabras clave: Enfermería; sindicatos; sociedades; docentes; ética en enfermería; Chile.

\section{ABSTRACT}

Introduction: The participation with the College of Nurses is a relevant action to support the development of nursing in Chile because this organization stands as an important defender to the practice. Therefore, it is advisable that nursing teachers promote this vision among the nursing community.

Materials and Methods: This is a quantitative, transectional and correlational study with 49 academicians from diverse teaching centers in the city of Puerto Montt, Chile, who were assessed in terms of their affiliation to the College of Nurses, their knowledge, attitudes, and motivations regarding their participation with this organization. A locally-designed instrument ( $\alpha$ Cronbach 0.71) was used.

Results: $40.9 \%$ of the teachers were affiliated to the College of Nurses. Associations between attitudes and working dynamics (Spearman Correlation $=0.158, p=0.043$ ), and affiliation to the College (Spearman Correlation $=0.142, \mathrm{p}=0.02$ ) were found .

Discussion: The participation with the College of Nurses can allow the strengthening of the profession by favoring its visibility, leadership, and professional identity, though these important gains are not always sufficient to encourage an affiliation or a positive attitude towards this organization.

Conclusions: In order to better promote the affiliation to the College of Nurses, young teachers' indifference attitudes, as well as other overall motivation factors should be considered first.

Keywords: Nursing; labor unions; societies; faculty; ethics nursing; Chile.

\section{RESUMO}

Introdução: A participação no colégio de enfermeiras é uma instancia relevante para o desenvolvimento da profissão ao nível do país, devido à defesa que realiza pela enfermagem desde os diferentes âmbitos de atuação; é indispensável inculcar esta visão desde a formação universitária a través dos profissionais de enfermagem que se dedicam à docência.

Materiais e métodos: Realizou-se um estudo quantitativo, transecional e correlacional, em 49 docentes das diferentes casas de estudo da cidade de Puerto Montt-Chile, em quem se avaliou sua filiação ao colégio de enfermeiras, além de seus conhecimentos, atitudes e motivações referentes a participar na instância gremial; para tal fim aplicou-se um instrumento de elaboração própria ( $\alpha$ Cronbach 0.71). Resultados: Encontrou-se que o 40.9\% dos docentes forma parte do colégio de enfermeiras e que existe conhecimento suficiente ao respeito desta organização, com uma atitude desfavorável a ele mesmo, evidenciando-se uma 
associação entre a atitude e aspectos como a dinâmica de trabalho ( $p=0.043$ Correlação de Spearman: 0.158) e a filiação ao colégio ( $p=0.02$ Coeficiente de Spearman: 0.142).

Discussão: A participação colegiada permite o fortalecimento da profissão, além de favorecer a visibilidade, liderança e identidade profissional; ainda que se reconheçam estes aspectos, não são suficientes para gerar a filiação ou uma atitude favorável a esta.

Conclusões: Na promoção da filiação deve considerar-se a tendência à não filiação dos docentes mais jovens e os aspectos de motivação, ambos relevantes para salientar a participação colegial como um aspecto deontológico que forma parte da modelagem aos enfermeiros em formação.

Palavras chave: Enfermagem; união; sociedades; docentes; ética de emfermagem; Chile.

\section{INTRODUCCIÓN}

Las organizaciones y asociaciones de enfermería se encuentran entre los medios más eficaces para el logro del bienestar y la promoción profesional, ya que apoyan a sus miembros e influyen en las políticas de crecimiento vertical del gremio, lo que se refleja en la alta calidad en la atención de enfermería ofrecida a los usuarios.

A nivel latinoamericano existen, agrupaciones profesionales de enfermería bajo el nombre de colegios profesionales o asociaciones nacionales, las cuales ejercen la defensa del gremio, labor trascendente si se tienen en consideración las dificultades para el ejercicio profesional que se vivencia en diferentes escenarios del cuidado de enfermería, y se hacen patentes en aspectos como la falta de insumos, entornos inapropiados que conducen a la sobrecarga laboral, pérdida de visibilidad y reconocimiento del profesional de enfermería por parte de los demás profesionales sanitarios, entre otros²,

En Chile, durante los últimos años se han advertido diversos niveles de agresión, cuando el colectivo de enfermería solicita reconocimiento en áreas profesionales en las que históricamente se ha desempeñado, por ejemplo, el área neonatal; o en su rol como profesión encargada de la Gestión de Cuidado4, aspecto reconocido por el código sanitario desde 19685, que ha generado la creación de subdirecciones de gestión del cuidado en los hospitales.

A manera de ejemplo de dichas agresiones, se encuentra el caso del Hospital Puerto Montt, ubicado en la Región de Los Lagos, donde los abusos de poder conllevaron a mantener la figura de la subdirección de gestión del cuidado, pero sin una estructura orgánica para facilitar la toma de decisiones y la administración del recurso humano de enfermería. Estos aspectos fueron debatidos y expuestos a la luz pública gracias a la acción de las organizaciones colegiales de enfermería como el Colegio de Enfermeras de Chile, encargado de velar por la defensa de la dignidad profesional.

Este colegio profesional, creado en 1953 con personalidad jurídica de derecho público (Ley 11.161); experimentó una transformación gradual hacia asociación gremial en 1981 debido al decreto de ley 3621, el cual suprimió la figura de colegio como corporación de derecho público, aspecto que volvió voluntaria la afiliación por parte de los profesionales de enfermería ${ }^{6,7}$.

En la actualidad, esta institución se define como una instancia gremial que defiende, protege y salvaguarda el ejercicio legal de la profesión; promueve entre sus asociados: la capacitación, defiende la exclusividad universitaria, el desarrollo de las subdirecciones de Gestión del cuidado, y mejores condiciones laborales, entre otros aspectos.

Pese a que la afiliación es de carácter voluntario, la participación en esta organización es considerada un deber ético del profesional de enfermería; aspecto que se encuentra consagrado en el código 
deontológico en el ámbito nacional ${ }^{8}$. Sin embargo; la escasa contribución por parte de los profesionales de enfermería, ubica a la disciplina en una situación de vulnerabilidad.

Desde la producción científica en enfermería, se aprecia insuficiente abordaje de esta temática en el ámbito nacional; no obstante, existe literatura científica con temáticas que guardan relación, como: las relaciones de poder, empoderamiento y participación de enfermería en entidades organizadas ${ }^{9,10}$.

El predominio de la investigación disciplinar en enfermería se centra principalmente en el área asistencial, educativa y administrativa o de gestión, dejando de lado su rol en la participación política ó gremial, aspecto que favorece la invisibilidad de la acción en dicho ámbito, y a su vez, la falta de reconocimiento por parte del entorno y de los mismos profesionales en lo concerniente a su liderazgo ${ }^{11}$.

Cabe mencionar que parte de las obligaciones de los profesionales de enfermería guardan relación con procurar el bienestar y dignidad profesional ${ }^{8}$. Para ello, es pertinente la participación significativa en instancias de su organización colegial, actividad que debe desarrollarse desde cualquiera de los ámbitos en los que se desempeña, siendo el rol docente el foco del presente trabajo debido a la importancia que tiene al forjar el modelaje de los enfermeros en formación ${ }^{12}$.

En consideración de lo anteriormente expuesto, se identificó la necesidad de analizar los conocimientos, actitudes y motivaciones respecto a la afiliación al colegio de enfermeras en profesionales de enfermería que se desempeñan como docentes en las Universidades de la ciudad de Puerto Montt-Chile, aspecto que constituye el objetivo general de la presente propuesta de investigación. Se establecen como objetivos específicos: establecer el perfil de los participantes, su nivel de conocimientos respecto de los estatutos del colegio de enfermeras, sus actitudes hacia dicha entidad, las motivaciones que tuvieron para colegiarse o que tienen para no hacerlo y, finalmente, analizar la asociación entre la colegiatura y las demás variables identificadas.

Se espera que los resultados de la investigación favorezcan el desarrollo de capacitaciones contextualizadas a las necesidades de la población en estudio y, a su vez, estimulen la generación de investigaciones sobre este tipo de temáticas a nivel latinoamericano, de forma que faciliten una aproximación al conocimiento de la realidad que se vivencia en los diferentes países.

\section{MATERIAL Y MÉTODOS}

La investigación se desarrolló bajo un diseño cuantitativo, transeccional y con análisis correlacional. Se consideró al universo de profesionales de enfermería que realizan actividades docentes en las universidades ubicadas en la ciudad de Puerto Montt - Región de Los Lagos, Chile a saber: Universidad de Los Lagos, Universidad Austral de Chile, Universidad San Sebastián, Universidad Santo Tomás. En total, lo constituían 65 docentes, a quienes se aplicaron criterios de inclusión y exclusión; se obtuvo una muestra de 49, considerada de tipo intencionado.

Los criterios de inclusión fueron docentes que: trabajen en universidades con acreditación institucional; con contratación vigente que laboren en las universidades ubicadas en la ciudad de Puerto Montt, independiente de su modalidad de contratación y de su afiliación al colegio de enfermeras; acepten su participación a través del proceso de consentimiento informado.

Los criterios de exclusión fueron docentes que: se encuentren en licencia, permisos, comisiones o en periodo sabático; sean parte del grupo que desarrolla la investigación o de la directiva regional y/o nacional del colegio de enfermeras.

Se tuvo en consideración indicar a los docentes que trabajan en diferentes universidades, responder sólo en aquella donde tenga el mayor número de horas contratadas. 
Tras la aprobación de las directivas de las universidades se procedió a realizar el proceso de consentimiento informado, el cual concluyó con la firma del acta de consentimiento.

El instrumento de recolección de información se elaboró a partir de las causas identificadas que han afectado la afiliación, según las apreciaciones de la directiva del Colegio de Enfermeras en la regional Puerto Montt. Posterior a su elaboración, fue sometido a juicio de expertos, quienes recomendaron: tener presente las modalidades de contratación existentes en las universidades, debido a la existencia de instituciones públicas y privadas, además de mantener las cinco opciones de respuesta para aquellas con escala Likert. La prueba de fiabilidad mediante alfa de Cronbach fue de 0.71.

Para la recolección de la información se mantuvo el anonimato de los participantes; no obstante, se identificó cada encuesta con un número consecutivo, con el fin de facilitar la organización de la información por parte de los investigadores.

El instrumento incluye IV secciones:

Sección I: Perfil del docente. Esta sección constó de preguntas para selección única de respuesta con posibilidad de ampliación, en aspectos como: sexo, edad, tiempo de experiencia laboral, último grado académico alcanzado, jerarquía académica, modalidad de trabajo, dinámica laboral; si se encuentra afiliado o no, y el tiempo de afiliación. Es de mencionar que la variable edad se reorganizó en grupos etarios para facilitar su interpretación.

Sección II: Conocimientos respecto al colegio de enfermeras. Para el abordaje de esta sección, se plantearon 24 afirmaciones basadas en el estatuto del Colegio de Enfermeras de Chile de 2017, con opciones de respuesta: sí, no, y no sé. Se asignó un puntaje de 1 a las afirmaciones cuya respuesta es un sí, excepto para tres de ellas (5, 8 y 16) cuya respuesta correcta era no. El puntaje se interpretó de la siguiente manera: Deficiente cuando el puntaje era menor de 14 puntos (menos del 60\%); suficiente para puntajes entre 14 y 19 puntos (6o-79\%) y sobresaliente para un puntuaje igual o mayor a 20 (más de 79\%). Cabe destacar que en esta sección se evaluaron 4 aspectos: conocimientos generales, actividades que realiza el colegio, requisitos para pertenecer al mismo, y dinámicas de funcionamiento.

Para la interpretación general de esta variable, se consideró que los puntajes de suficiente y sobresaliente correspondían a la categoría conoce, mientras que el de deficiente se estableció como desconoce.

Sección III: Actitudes respecto a la colegiatura. Comprendió 9 afirmaciones en escala tipo Likert con 5 opciones de respuesta: totalmente de acuerdo, de acuerdo, ni en acuerdo ni en desacuerdo, en desacuerdo y totalmente en desacuerdo. Para la interpretación de la escala se tuvo en cuenta un valor mínimo de 9 y máximo de 45 puntos, con respecto a la clasificación se eligieron los siguientes rangos: muy baja de 9 a 17 puntos, baja de 18 a 26 puntos, media de 27 a 35 puntos y alta de 36 a 45 puntos. En la interpretación general, se consideró la clasificación de alta como una actitud favorable respecto a la colegiatura y desfavorable las demás categorías; se incluyó la categoría media en esta última clasificación, debido a la correspondencia deontológica que representa el tener una actitud favorable hacia la colegiatura.

Sección IV: Motivaciones. Para identificar las motivaciones, se plantearon preguntas diferenciadas para los miembros de un colegio y para los que no pertenecen a uno; se les preguntó cuáles fueron los motivos para afiliarse, colegiarse o para mantenerse sin membresía respectivamente, dando opciones de respuesta múltiple con posibilidad de ampliación bajo el criterio de otras. 
Las variables principales de valoración fueron aquellas pertenecientes al perfil de los docentes, además de sus conocimientos, actitudes y motivaciones.

Los datos se capturaron en Excel ${ }^{\circledR}$, para posteriormente ser procesados en el software SPSS versión 20. Para el componente descriptivo, se emplearon frecuencias y porcentajes; en el correlacional, se aplicó la prueba Chi cuadrado y coeficiente de correlación de Spearman, considerándose como asociación significativa cuando las variables presentaban un $\mathrm{Chi}^{2} \mathrm{p}=\leq 0.05$.

El trabajo se desarrolló según lo establecido en la legislación vigente a nivel país, principalmente por las leyes de investigación 20.120 y de protección de la vida privada 19.628, las pautas éticas de la CIOMS (Council for International Organizations of Medical Sciences-CIOMS-OMS), los criterios de Ezequiel Emanuel, y otras normativas internacionales relacionadas ${ }^{2,13-15}$. Se contó con la aprobación del Comité ético científico del servicio de salud del Reloncaví mediante ordinario 53-2018.

\section{RESULTADOS}

Para dar cumplimiento a los objetivos, en primer lugar, se estableció el perfil de los participantes, se identificó que la mayoría son de sexo femenino, se encuentran en etapa de adulto medio y más de la mitad (65.3\%) tiene 6 años o más de experiencia laboral. En cuanto al último grado académico alcanzado, una gran parte posee sólo el título profesional y el 22.5\% tiene formación de magíster; el nivel de doctorado no se evidenció. Es de destacar que el 51\% de los profesionales participantes no posee jerarquía académica (Tabla 1).

En relación con la modalidad de trabajo, el 57.1\% tiene contratos a término fijo u honorario, reflejan inestabilidad en la relación contractual y dinámica laboral; dos terceras partes se dedican exclusivamente a la docencia (65.3\%). En este grupo un poco más de la mitad posee contrato indefinido (56.2\%); el porcentaje restante se encuentra por honorarios o con contratos a término fijo (Tabla 1).

Respecto de la afiliación al colegio de enfermeras, se encontró que el 59.1\% no forma parte de uno, mientras seis de ellos (12.2\%), se encuentran afiliados a otras asociaciones gremiales de salud o propias de las casas de estudio.

En el segundo objetivo de la investigación se indagó respecto al conocimiento acerca del colegio de enfermeras, ante lo cual se pudo identificar que el 73.5\% tiene conocimientos entre suficiente y sobresaliente, y el $26.5 \%$ conocimientos deficientes. Al analizar cada uno de los ítems evaluados en el conocimiento, se pudo apreciar que el de actividades que realiza el colegio es el mejor evaluado, con un $68.5 \%$, seguido de los aspectos generales, los requisitos para pertenecer al mismo y sus dinámicas de funcionamiento con un 56.1\%, 55.6\%, y 52.5\% respectivamente (Gráfica 1).

Al evaluar en forma específica los componentes de cada ítem, se aprecia que la mayoría de los participantes identifican de forma correcta que, entre las labores del colegio, se encuentra velar por el prestigio de la profesión y los asociados (91.8\%); no obstante, en las generalidades, el 55.1\% desconoce que la afiliación es parte de los aspectos que contempla el código de ética de enfermería a nivel país.

La relación entre el conocimiento y la afiliación, refleja tendencia hacía el desconocimiento en los no afiliados; y alcanza un 34\%, en comparación con los afiliados; para estos últimos el desconocimiento fue de $15 \%$.

El tercer objetivo de la investigación fue establecer las actitudes de los participantes hacia el colegio de enfermeras; se destaca el predominio de la actitud desfavorable, tanto en colegiados como en no colegiados (alrededor del 90\%). 
Al evaluar cada parámetro por el cual se determinó la actitud, se pudo apreciar que los participantes identificaron favorablemente que esta instancia de participación gremial, propende a fortalecer aspectos relacionados con la dignidad y la identidad profesional. Entre las actitudes identificadas

Tabla 1. Perfil de los participantes $(n=49)$

\begin{tabular}{lc}
\hline Variable & $\%$ \\
\hline Sexo & \\
\hline Masculino & 12.2 \\
\hline Femenino & 87.8 \\
\hline Grupo etario & 32.7 \\
\hline Adulto joven (20 a 29 años) & 65.3 \\
\hline Adulto Medio (3o a 64 años) & 2.0 \\
\hline Adulto Mayor (65 años y más) & 2.0 \\
\hline Experiencia laboral & 32.7 \\
\hline Menos de un año & 22.4 \\
\hline 1 a 5 años & 24.5 \\
\hline 6 a 1o años & 18.4 \\
\hline 11 a 2o años & 77.5 \\
\hline Más de 20 años & 22.5 \\
\hline Formación profesional y de postgrado & \\
\hline Título profesional & 49.0 \\
\hline Título profesional y magíster & 51.0 \\
\hline Jerarquía académica & 36.1 \\
\hline Instructor/a, asistente, asociada/o, titular & 20.4 \\
\hline Sin jerarquía & 36.7 \\
\hline Modalidad de trabajo & \\
\hline Contrato a término indefinido & \\
\hline Contrato a término fijo & \\
\hline Honorario & \\
\hline Dinámica de trabajo & \\
\hline Dedicación total a la docencia & \\
\hline Dedicación parcial (Hospital, Centro de salud u otros) & \\
\hline Afiliación al colegio de enfermeras & \\
\hline Si & \\
\hline No & \\
\hline & \\
\hline
\end{tabular}

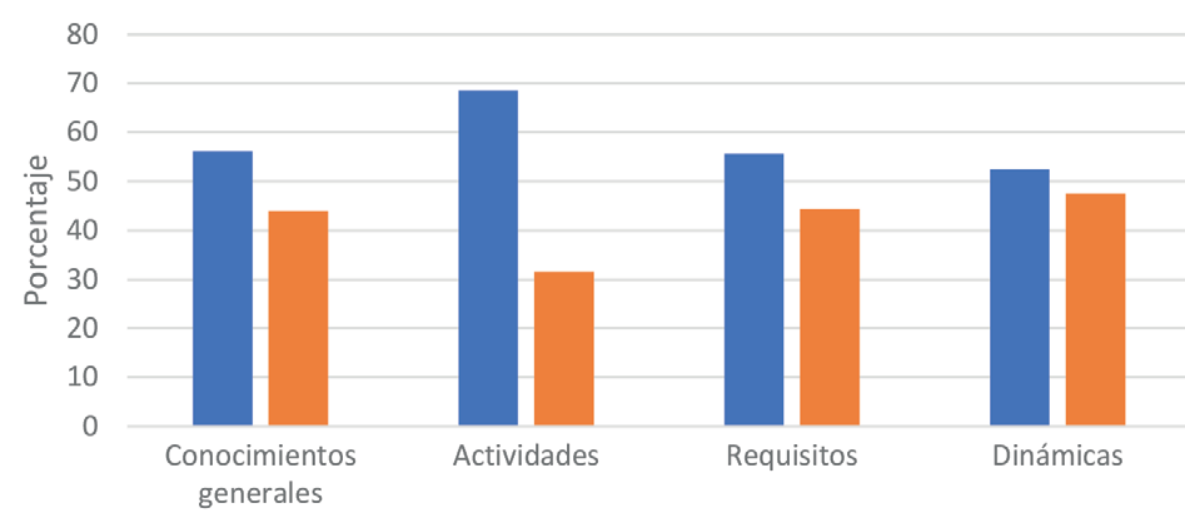

$\square \mathrm{SI}=\mathrm{NO}$

Gráfica 1. Conocimientos respecto del Colegio de enfermeras $(n=49)$ 
como desfavorables, se visualizan aspectos de escasa proactividad en cuanto a la obtención de información respecto al colegio de enfermeras y la participación en actividades en las cuales se esperaría desarrollar procesos dialógicos constructivos hacia su funcionamiento.

Como cuarto objetivo, la investigación fue dirigida a conocer las motivaciones que tuvieron los colegiados para afiliarse al colegio de enfermeras, y las que, en forma contrapuesta, tienen los no colegiados para mantener dicha condición.

Se logró identificar en la población de colegiados, que el principal motivo fue el fortalecer las instancias de participación profesional y contar con el apoyo del colegio ante situaciones laborales en que se requiera (85\%). En los individuos no colegiados, se aprecia que desconocer la utilidad de estar colegiado (24.1\%) y cómo hacer los trámites de afiliación (24\%) fueron los principales aspectos (Tabla 2).

En el parámetro de otros, los participantes refirieron aspectos como: en los años que empecé era complicado pertenecer a organizaciones que no fuera tu lugar de trabajo y diferentes combinaciones entre las opciones que se dieron; las más notables: Considero que las directivas regionales no me representan, Desconozco cuál sería la utilidad de estar colegiada/o y El costo mensual de la colegiatura.

Para dar cumplimiento al quinto y último objetivo, se realizó el análisis correlacional de las variables, a partir del cual se pudo establecer significancia estadística entre la variable actitud y las variables del perfil: dinámica de trabajo ( $p=0.043$ Correlación de Spearman: 0.158) y afiliación al colegio

Tabla 2. Motivaciones para afiliarse o mantener la no afiliación al colegio de enfermeras

\begin{tabular}{lr}
\hline Motivaciones & $\%$ \\
\hline Colegiados $(n=20)$ & 85.0 \\
\hline $\begin{array}{l}\text { Fortalecer las instancias de participación profesional y contar con el apoyo y/o contar con el apoyo } \\
\text { del colegio ante cualquier situación laboral en la que así se requiera. }\end{array}$ & 15.0 \\
\hline Sentí presión de mis colegas y/o de la institución en la trabajo para hacerlo. & 13.8 \\
\hline No colegiados ( $n=29)$ & 24.1 \\
\hline El costo mensual de la colegiatura & 6.9 \\
\hline Desconozco cuál sería la utilidad de estar colegiada/o & 24.0 \\
\hline Considero que las directivas regionales no me representan. & 6.9 \\
\hline Desconozco cómo hacer los trámites de afiliación & 24.1 \\
\hline He solicitado la colegiatura anteriormente, pero no he obtenido respuesta.
\end{tabular}

Tabla 3. Análisis correlacional entre el perfil, conocimiento y actitud

\begin{tabular}{lcccc}
\hline \multirow{2}{*}{ Variables del perfil } & \multicolumn{3}{c}{ Conocimiento } & Actitud \\
\cline { 2 - 5 } & $\mathrm{p}$ & Correlación de Spearman & $\mathrm{p}$ & Correlación de Spearman \\
\hline Grupo etario & .150 & -- & .228 & -- \\
\hline Sexo & .687 & -- & .378 & -- \\
\hline Experiencia laboral & .343 & -- & .306 & -- \\
\hline Grado académico & .144 & -- & .393 & -- \\
\hline Jerarquía académica & .355 & -- & .605 & -- \\
\hline Modalidad de trabajo & .696 & -- & .374 & -- \\
\hline Dinámica de trabajo & .737 & -- & .043 & .158 \\
\hline Afiliación al colegio & .129 & -- & .002 & .142 \\
\hline Tiempo de colegiatura & .356 & -- & .854 & -- \\
\hline
\end{tabular}


( $p=0.02$ Coeficiente de Spearman: 0.142), motivo por el cual se puede mencionar que la actitud mostrada se asocia con aquellos docentes que se dedican exclusivamente a la docencia y aquellos que no pertenecen al colegio de enfermeras (Tabla 3).

Pese a que se evidencia en forma descriptiva la tendencia al desconocimiento en la población no afiliada, ésta no tuvo alguna significancia estadística en el análisis correlacional, al igual que entre el conocimiento y las demás variables del perfil ( $p \geq 0.05$ ) (Tabla 3).

Al analizar la afiliación al colegio de enfermeras en relación con las variables del perfil, se observa asociación de tipo negativo entre ésta y el grupo etario ( $p=0.040$ Correlación de Spearman -0.335); se distingue una menor afiliación entre la población de docentes más jóvenes (Tabla 4).

Tabla 4. Análisis correlacional entre el perfil y la colegiatura

\begin{tabular}{lcc}
\hline \multirow{2}{*}{ Variables del perfil } & \multicolumn{3}{c}{ Colegiatura } \\
\cline { 2 - 3 } & $\mathrm{p}$ & Correlación de Spearman \\
\hline Grupo etario & .040 & -.335 \\
\hline Sexo & .691 & - \\
\hline Experiencia laboral & .312 & - \\
\hline Grado académico & .217 & - \\
\hline Jerarquía académica & .181 & -- \\
\hline Modalidad de trabajo & .367 & \\
\hline Dinámica de trabajo & .548 & \\
\hline
\end{tabular}

\section{DISCUSIÓN}

Respecto al perfil sociodemográfico, puede mencionarse que coincide con el reportado en otros estudios realizados en docentes universitarios de enfermería, en el cual se aprecia predominio de mujeres y la etapa de adulto medio ${ }^{16-18}$. El hallazgo respecto a la menor afiliación en docentes jóvenes es un aspecto relevante a considerar; sí se tiene presente que, según datos del Ministerio de Salud de Chile, en el 2016 aproximadamente el 69.9\% de la población laboral en enfermería tenía menos de 45 años ${ }^{19}$.

En cuanto al perfil laboral, la mayoría de los docentes no posee jerarquía académica, proceso que permite establecer carrera académica en relación con su producción intelectual, tiempo de ejercicio docente, entre otros aspectos que varían en cada institución universitaria. La ausencia de este parámetro es un factor que va en detrimento de las posibilidades de mejora de sus condiciones a futuro, a las que se suma la inestabilidad en sus condiciones de trabajo.

Tanto la falta de jerarquización como la modalidad de contratación representan inestabilidad laboral y mayor riesgo de afección psicoemocional y social; aspectos que se hacen notar como una problemática actual en el desempeño de otros roles del profesional de enfermería ${ }^{20,21}$.

En el abordaje de los aspectos relativos al colegio de enfermeras, el conocimiento que los docentes tienen respecto al funcionamiento del mismo, según sus estatutos se encuentra en un nivel destacado, en contraste con su actitud desfavorable y bajo porcentaje de afiliación a esta instancia de participación gremial. Estos aspectos son importantes desde el punto de vista de la docencia, ya que si bien el primero permitiría su incorporación en el proceso de enseñanza-aprendizaje, los otros dos podrían considerarse como parámetros que se transmiten en el modelaje que tiene el docente hacia los estudiantes, y representa un factor que va en detrimento de estrategias que propenden por la visibilidad, el liderazgo y la consolidación de la identidad profesional ${ }^{22-28}$. Debe considerarse que, 
de acuerdo con los hallazgos, el promover y garantizar la afiliación al colegio de enfermeras puede ser una estrategia que favorezca la actitud favorable hacia el mismo.

La tendencia hacia la no afiliación en docentes más jóvenes y los hallazgos alusivos a las motivaciones, son aspectos que deben considerarse al momento de promover la afiliación en este grupo de profesionales, destacar la importancia que tiene su participación en instancias políticas como parte del modelaje relacionado con el sentido de pertenencia hacia la profesión y el liderazgo, los cuales se forjan durante la trayectoria formativa de los estudiantes, para contribuir con la disminución de las barreras existentes para el posicionamiento político del profesional de enfermería en el contexto laboral actual ${ }^{29}$.

Es de mencionar que las motivaciones detectadas para no estar afiliado al colegio son fácilmente superables a través de procesos efectivos de comunicación y difusión; lamentablemente la literatura científica disponible respecto a esta temática dificultó contrastar los resultados obtenidos en este aspecto.

Debido a la metodología del estudio, se pudo hacer un análisis cuantitativo que permitió detectar asociación entre algunas variables, sin que ello represente una relación de causalidad. Se debe tener precaución al interpretar y pretender la generalización de los resultados en consideración, que corresponde a un contexto particular. Destaca la necesidad de desarrollar investigaciones cualitativas que permitan indagar a fondo los hallazgos establecidos en esta investigación, motivo por el cual se reconoce que estos aspectos configuran las limitaciones de la presente investigación.

\section{CONCLUSIONES}

La afiliación a los colegios y/o asociaciones profesionales de enfermería puede considerarse como un aspecto deontológico y, por lo tanto, indispensable, que cobra mayor importancia en aquellos que realizan labores docentes debido al modelaje que se transmite hacia sus estudiantes, quienes requieren, más allá de la formación de aspectos científico- técnicos; visualizar en sus docentes actitudes de participación política, que serán de utilidad para el desarrollo del pensamiento crítico y el liderazgo, competencias clave para el fortalecimiento de un ejercicio profesional que permita el posicionamiento de la enfermería en sus diferentes ámbitos de acción.

Se puede considerar necesario fomentar entre los enfermeros dedicados a la academia, la afiliación hacia las instancias de participación gremial como lo es el colegio de enfermeras, con lo cual existe mayor probabilidad de forjar actitudes favorecedoras hacia el mismo. Un foco importante es el acompañamiento por parte de las académicas/os con mayor experiencia hacia las/os más jóvenes, en quienes se evidenció menor participación.

\section{RESPONSABILIDADES ÉTICAS}

Protección de personas y animales. Se declara que en este estudio no se realizó algún tipo de experimentación en personas ni animales.

Confidencialidad de datos. Se declara que en el artículo no se emplean datos de pacientes ni de los participantes que permitan su identificación.

Derecho a la privacidad y consentimiento informado. Los autores declaran que estos aspectos fueron resguardados de acuerdo con la legislación vigente en el país y las normativas éticas internacionales de investigación, contando con el aval del comité ético científico acreditado de la región.

Financiamiento: Ninguno. 
Conflicto de interés: Los autores declaran no tener conflictos de interés.

Los autores agradecen a las directivas de las casas de estudios por permitir el desarrollo de la investigación y a las directivas del colegio de enfermeras regional Puerto Montt, por acoger esta iniciativa.

\section{REFERENCIAS}

1. Esmaeili M, Dehghan-Nayeri N, Negarandeh R. Factors impacting membership and non-membership in nursing associations: a quialitative Study. Nurs Health Sci 2013; 15(13): 265-72 http://dx.doi:10.1111/nhs.12012

2. Canales-Vergara M, Valenzuela-Suazo S, Paravic-Klijn T. Condiciones de trabajo de los profesionales de enfermería en Chile. Enferm. Univ. 2016; 13(3): 178-86. http://dx.doi.org/10.1016/j.reu.2016.05.004

3. Aspiazu EL. Las condiciones laborales de las y los enfermeros en Argentina: entre la profesionalización y la precariedad del cuidado en la salud. Trab. Soc. 2016; 28: 11-35.

4. Canal 5 Chile. Enfermeros y enfermeras del Hospital Puerto Montt se encuentran en Paro [video file] 2016-jun-24. [2:10 min]. https://www.youtube.com/watch?v=-PpKZ_iGzgk

5. Ministerio de Salud Pública. Código Sanitario. Decreto con fuerza de Ley No. 725. Santiago: MinSalud; 1968. Última Versión: 2 de Mayo de 2019: Ley 21155. https://bit.ly/2V5dwGO

6. Paredes MP, Rivas RE. Historia del ejercicio profesional de enfermeras hospitalarias del sur de Chile (1940-1980). Cienc. Enferm 2014; 2O(1): 9-21. https://dx.doi.org/10.4067/So717-95532014000100002

7. Colegio de Enfermeras. Reseña cronológica de la Enfermería en Chile 1902-2006. Chile: Odonto Info. 2017-diciembre-25. https://bit.ly/2SVUPm8

8. Colegio de Enfermeras de Chile. Código de ética del Colegio de Enfermeras de Chile. Santiago: Colegio de enfermeras de Chile; 2008. 17p. https://bit.ly/37zPNB5

9. Chuaqui-Kettlun JR, Bettancourt-Ortega LP, Leal-Román VJ, Aguirre-González CA. La identidad profesional de la enfermería: un análisis cualitativo de la enfermería en Valparaíso (1933-2010). Aquichan 2014; 14(1): 53-66. https://bit.ly/2SO-saPn

10. Cotos Alva RM. Reflexión: desidia e indiferencia del profesional de enfermería en el desarrollo de la función administrativa y gerencial. In Cres CS 2018; 4(2): 625-9.

11. Quezada Torres C, Illesca Pretty M, Cabezas González M. Percepción del liderazgo en las(os) enfermeras(os) de un hospital del sur de Chile. Cienc. enferm 2014; 20(2): 41-51. http://dx.doi.org/10.4067/So717-95532014000200005

12. Rojas Valenciano LP. El fenómeno del poder en enfermería en la academia y el área asistencial desde la perspectiva del docente. Horizonte Sanitario 2019;18(1): 17-26.

http://dx.doi.org/10.19136/hs.a18n1.2067

13. Bachelet Jeria M. Ley n 20120: sobre la investigación científica en el ser humano, su genoma, y prohibe la clonación humana. Rev. chil. obstet. ginecol. 2007; 72(2): 133-5.

http://dx.doi.org/10.4067/So717-75262007000200013

14. Secretaría General de la presidencia. Ley 19.628. Sobre la protección de la vida privada. Santiago: Secretaría General de la Presidencia. última versión: 23 Julio 2011.

15. Organización Panamericana de la Salud y Consejo de Organizaciones Internacionales de las Ciencias Médicas. Pautas éticas internacionales para la investigación relacionada con la salud con los seres humanos. $4^{a}$ ed. Ginebra: Consejo de Organizaciones Internacionales de las Ciencias Médicas (CIOMS); 2016.150 p.

16. Álvarez-Viera P. Ética e investigación. bol. redipe 2018; 7(2): 122-49. https://bit.ly/37K34Y9 
17. Veiga Madriaga LC, Dantas de Oliveira Souza NV, Arantes Ferreira Brecht D'Oliveira C, Carneiro Carvalho E, Luz Lisboa MT, Silva de Andrade KB. O docente de enfermagem: uma análise sociodemográfica, laboral e de saúde. Rev enferm UFPE on line 2019; 13(2): 438-48.

18. Triviño-Vargas ZG, López-Hurtado MX. Factores predictores de conductas promotoras de salud en docentes de enfermería de tres universidades de Cali, Colombia. Univ. Salud 2018; 20(2): 160-70. http://dx.doi.org/10.22267/rus.182002.120.

19. Ministerio de salud de Chile. Informe de brechas de personal de salud por servicio de salud. Santiago: Subsecretaría de redes asistenciales; 2017, 89p. https://bit.ly/2uXpirZ

20. Draganov PB, Sanna MC. Competencias Andragógicas de los docentes enfermeros que actúan en la graduación en enfermería paulistana. Trab. educ. saúde 2016; 14(1): 155-82. http://dx.doi.org/10.159o/1981-7746-sipooog8

21. Cogollo Milanés Z, Gómez Bustamante E. Condiciones laborales en enfermeras de Cartagena, Colombia. Av. Enferm. 2010; 28(1): 31-38. https://bit.ly/2P6OStP

22. Contreras A, Galindo AK, García N, Villalpando EM, Godinez MA. Construcción de la identidad profesional en los estudiantes de la licenciatura en enfermería. Cuidarte. 2019; 8(15): 80-95 http://dx.doi.org/10.22201/fesi.23958979e.2019.8.15.69159

23. Ruíz-Carrillo E, Suárez-Castillo P, Cruz-González JL, López-Ríos JD. Análisis discursivo del docente en la formación del profesional de enfermería: el cuidado de los pacientes. REPI Revista electrónica de Piscología Iztacala 2017; 20(3): 872-84. https://www.medigraphic.com/pdfs/epsicologia/epi-2017/epi171e.pdf

24. Vera M, Cornejo R, Rivas L, Calizaya J, Zamora O, Garcia J. Significado de los valores profesionales en docentes y estudiantes de enfermería, UNMSM - 2014. An. Fac. Med., Univ. Nac. Mayor San Marcos. 2016; 77(3): 225-9. https://bit.ly/326oev6

25. Moscoso P. Calidad de la docencia en enfermería: un concepto desde la percepción de sus actores. FEM 2013; 16(2): 97-104. http://dx.doi.org/10.4321/S2014-98322013000200008

26. Valerio-Ureña G, Rodríguez-Martínez MC. Perfil del profesor universitario desde la perspectiva del estudiante. Innov. Educ. 2017; 17(74): 109-24. https://bit.ly/39GeT2A

27. González-Aguilar A, Vásquez-Cataño F, Almazán-Tlalpan B, Morales-Nieto A, García- Solano B. Proceso de aprehensión de identidad profesional en enfermería. Rev Cuid 2018; 9(3): 2297-308. https://doi.org/10.15649/cuidarte.v9i3.519

28. Wood C. Nursing Now campaign: time to break your silence. Journal of Community Nursing 2018; 32(6): 14-6. https://bit.ly/2SUwzRr

29. Rutz Porto A, Buss Thofehrn M. El empoderamiento político de los enfermeros en la práctica hospitalaria. Index Enferm 2015; 24(1-2): 2O-24. http://dx.doi.org/10.4321/S1132-12962015000100005 